\title{
Six-Year-Old Sheep as a Clinically Relevant Large Animal Model for Aortic Valve Replacement Using Tissue-Engineered Grafts Based on Decellularized Allogenic Matrix
}

\author{
Karolina Theodoridis, DVM, ${ }^{1,2}$ Igor Tudorache, MD, ${ }^{1,2}$ Serghei Cebotari, MD,,2 \\ Alexandru Calistru, MD,,2 Tanja Meyer, DVM, ${ }^{1,2}$ Samir Sarikouch, MD, \\ Christoph Bara, MD, Axel Haverich, MD, ${ }^{1,2}$ and Andres Hilfiker, $\mathrm{PhD}^{1,2}$
}

\begin{abstract}
Tissue-engineered (TE) grafts based on decellularized grafts have shown very promising results in preclinical and clinical studies. However, in animal models valves have either been tested in juvenile models or in the clinically less relevant pulmonary valve position. In this study, we tested the grafts in the aortic valve (AV) position of 6-year-old sheep, as geriatric patients in need of an $\mathrm{AV}$ substitute due to calcification are the largest patient group benefiting from TE grafts. Decellularized AV (DAV; $n=4)$ and DAV additionally re-endothelialized with autologous cells $(n=3)$ were implanted in the AV position of 6-year-old female sheep. Function was investigated at implantation and explantation 12 months later. Regeneration capacity was analyzed by the repopulation degree of the graft with recipient's cells, by the generation of a new endothelial layer and by intracellular staining against pro-collagen type I. DAV and re-endothelialized AV demonstrated excellent function with only two valves developing mild insufficiencies $\left(1^{\circ}\right)$. Of the repopulating cells only few cells were identified as inflammation cells, while the majority was found to be interstitial cells producing procollagen type I. Endothelial coverage was found, but seemed to be reduced. The regenerative capacity of decellularized matrix is not only a feature exhibited when implanted in juvenile individuals but also is evident when implanted in the highpressure AV position of older sheep, revealing the potential of TE grafts in age-advanced patients.
\end{abstract}

Keywords: aged animal model, matrix guided tissue regeneration, decellularization, re-endothelialization

\section{Introduction}

$\mathbf{H}$ EART VALVE PROCEDURES are increasing in number, especially due to population aging which has resulted in rising prevalence of acquired heart diseases. Currently, the ideal substitute for heart valve replacement in patients, both young and old, remains elusive. However, therapeutic goals differ depending on the patient's age. While the survival rate and reduction in the overall number of operations is of highest interest for pediatric patients, improving the quality of life is the main focus for geriatric patients. ${ }^{1}$

Tissue-engineered (TE) allografts based on decellularized matrices may provide the ideal solution, as they have shown excellent long-term results compared to other biological grafts, with longer freedom from explantation, reduced immunological reaction, and even signs of adaptive growth in children. ${ }^{2,3}$ Preclinical and early clinical studies on decellularized allografts have so far focused on young individuals, and the observed repopulation of the grafts suggests a regeneration of the tissue, indicative of growth. Where growth is not a major objective, as, for example, in older patients, other valve replacement options are available.

Mechanical grafts have lifelong durability, but their poorer hemodynamic properties, leading to higher rates of bleeding as a result of the necessary anticoagulant therapy, represent clear drawbacks. ${ }^{4}$ Despite a higher rate of structural valve failure, bioprostheses have been favored as a graft for elderly patients, ${ }^{5}$ as they are associated with a higher life expectancy in 70-year-old patients. ${ }^{6}$

Life expectancy is increasing globally, and a calculation of data from 2010 revealed that the group of 85-year-olds in the US population is expected to triple in number by the

\footnotetext{
${ }^{1}$ Leibniz Research Laboratories for Biotechnology and Artificial Organs (LEBAO), Hannover Medical School, Hannover, Germany.

${ }^{2}$ Department of Cardiothoracic, Transplantation and Vascular Surgery, Hannover Medical School, Hannover, Germany.

*This article is part of a special focus issue on Animal Models in Tissue Engineering. Part II.
} 
year $2049^{7}$ with a corresponding rise in patients requiring heart valve procedures to be expected. The number of young patients, in contrast, has stagnated. A study conducted in the United States with 11,911 patients showed that the prevalence of valve disease among those over 75 is 13\%. In Europe, the mean age of patients with valvular heart diseases is $64 \pm 14$ years, and the number of patients receiving a bioprosthesis increases correspondingly with age. ${ }^{8}$ Despite the fact that elderly patients represent the largest share of those affected by valve-related diseases, very little is known to date about the regenerative potential of decellularized heart grafts in older individuals.

It is generally to be expected that cell repopulation of decellularized grafts is reduced in aged patients due to an overall reduction in regenerative potential. Possible physiological explanations for this loss of regeneration are the reduced number of stem cells and progenitor cells ${ }^{9}$ and the age-related shortening of telomeres. ${ }^{10}$ In fact, in a previous study examining the performance of TE pulmonary valves (PVs) based on decellularized matrices in 7-year-old sheep, we observed reduced repopulation with autologous cells compared to other studies undertaken in juvenile animals. ${ }^{11}$ However, repopulation with interstitial cells was observed in the valvular wall and in the cusp even in older sheep. The repopulation of grafts with interstitial autologous cells in older individuals may be considered circumstantial as somatic growth is complete. However, regeneration and self-remodeling remain important in later life, not only to overcome minor damage in organs but also as an instrumental factor in immunological reactions.

In previous studies, we have shown that ovine aortic valves (AVs) can be successfully decellularized and, when implanted in juvenile sheep, they show an improved outcome in comparison with cellular grafts. ${ }^{12}$ We also demonstrated that decellularized PVs are repopulated to a large degree in older sheep, but full repopulation was not achieved even when the grafts were previously re-endothelialized with autologous endothelial-like late outgrowth cells (endothelial cell [EC]). ${ }^{11}$ The PV replacement was used as a proof-of-concept method to examine whether EC can be generated from older individuals and if there is any evidence of repopulation. As defects of the PV are mainly congenital, the overwhelming majority of patients receiving a PV reconstruction are under 18 years of age. ${ }^{13}$ In contrast, the AV is the most frequently replaced heart valve, with the degeneration of the AV being the main cause in $81.9 \%$ of the cases. ${ }^{8}$

The aim of this study was to analyze the regenerative potential of decellularized grafts in the AV position in 6year-old sheep. This differs from previously published results concerning grafts implanted in the PV position in a number of ways, which lead to different repopulation and cell migration patterns. First, (1) the AV is subjected to significantly higher pressure than the PV, which is associated with more physical stress. Second, (2) the histoarchitecture is different in terms of the valvular wall thickness and protein composition. ${ }^{14}$ Finally, (3) there are differences in surgical approach in terms of the implantation site, which can also affect the clinical outcome. While the PV is implanted in a more distal position compared with the native valve in animal studies, the AV is implanted in the root position to allow the coronary ostia to be connected.

In this study, we implanted decellularized aortic valves (DAVs, $n=4$ ) and re-endothelialized decellularized aortic valves (EAVs, $n=4$ ) to analyze whether the endothelium is necessary to prevent thrombogenicity when implanted in the more challenging position of the AV. In the low-pressure environment of the PV, however, re-endothelialization did not have an observable influence on the function or repopulation of decellularized grafts. ${ }^{11}$

While previous studies include decellularized valves implanted in the PV position of elderly sheep and in the AV position of juvenile sheep, this is the first long-term study of decellularized valves conducted in the clinically most relevant position of the AV in age-advanced large animal model.

\section{Materials and Methods}

\section{Decellularization of $A V s$}

AVs were obtained at the local slaughterhouse from adult sheep. After transportation to the laboratory decellularization was conducted under sterile conditions as previously described. ${ }^{12}$ In brief, valves were decellularized in a detergent solution containing sodium dodecyl sulfate $(0.5 \%)$ and sodium deoxycholate $(0.5 \%)$ for $48 \mathrm{~h}$. Cell detritus and detergent were removed by washing with deionized water (24h) and phosphate buffered saline $(60 \mathrm{~h})$.

\section{Generation of late outgrowth ECs}

Mononuclear cells were isolated as published by Aper et al. ${ }^{15}$ One hundred milliliters blood was collected from the Vena jugularis of sheep. After centrifugation, the mononuclear phase was distributed in two $75 \mathrm{~cm}^{2}$ Nunc cell culture flask containing endothelial growth medium (EGM-2, Lonza) enriched with $10 \%$ fetal calf serum. Two to three weeks later first colonies of endothelial-like cells became visible. Cells were further passaged in a 1:3 manner until a uniform morphology and a sufficient number of cells were reached. For the experiments, ECs were used in passage 4-6. To distinguish seeded cells from cells repopulating the AV after implantation, ECs were transfected by lentivirus to express red fluorescent protein (RFP) as described previously. ${ }^{16}$

\section{Re-endothelialization of the DAVs}

The re-endothelialization of decellularized valves in the bioreactor of Lichtenberg has been described previously by this working group. ${ }^{17,18}$ After closing the coronary arteries and suturing the valves into the bioreactor, valves were seeded under rotating conditions with autologous ovine EC (per valve $11.3 \pm 1.0 \times 10^{6}$ ). The bioreactor was connected to a roller pump leading to a circulating flow through the valve (EGM-2 medium). The flow was increased gradually from $0.02 \mathrm{~L} / \mathrm{min}$ by $0.5 \mathrm{~L} / \mathrm{min}$ twice a day, until it reached $1 \mathrm{~L} /$ min. In the attached gas exchange chamber the circulation medium was oxygenized.

\section{Animal experiment}

The animal experiment was approved by the German authorities of Lower Saxony under the reference number 08/1570.

Female sheep $(n=8)$ received AV replacement using either a DAV $(n=4)$ or a DAV, which was re-endothelialized with autologous ovine EC before implantation (EAV, $n=4)$. At time point of implantation the sheep were 6 years of age, thus fully grown, and had an average weight of $80.3 \pm 10.0 \mathrm{~kg}$. 
After premedication with midazolam $(0.15 \mathrm{mg} / \mathrm{kg})$, and inducing anesthesia with Propofol ${ }^{\circledR}$, anesthesia was maintained by isoflurane (3-4\%). Following thoracotomy and attachment to the heart lung device, DAVs and EAVs were implanted as described previously as full root replacement of the AV, including reimplantation of the coronary vessels. ${ }^{19}$ After implantation the first functional analysis of the grafts was conducted under full anesthesia. By transesophageal echocardiography valve diameter, orifice area (using the continuation equation), ejection fraction, and gradient of the graft, as well as possible stenosis and insufficiency, were measured.

Analgesic treatment was given to all sheep during implantation (Fentanyl, $0.01 \mathrm{mg} / \mathrm{kg}$ ) and in the first 4 days after surgery (carprofen $4 \mathrm{mg} / \mathrm{kg}$, and buprenorphine $0.01 \mathrm{mg} / \mathrm{kg}$, subcutaneous [s.c.]). Antibiotic treatment was administered for 4 days (penicillin $92 \mathrm{mg} / \mathrm{kg}$ and streptomycin $80 \mathrm{mg} / \mathrm{kg}$, s.c.) and antithrombotic prophylaxis for 14 days (enoxaparinsodium $1 \mathrm{mg} / \mathrm{kg}$, s.c.).

Twelve months after implantation and after conducting a second transesophageal echocardiographical examination, animals were euthanized under full anesthesia by injecting pentobarbital (intravenous). After death the heart, including the aortic graft, was explanted and processed for further analysis.

\section{Evaluation of the cusps' surface repopulation}

Half a cusp of each graft was stained in toto with phalloidin and 4',6-diamidino-2-phenylindole (DAPI). While DAPI stains nuclei, phalloidin stains the cytoskeleton of cells and was used to calculate the percentage of the arterial and ventricular cusp surface covered with cells, in relation to the complete surface area, after taking a MosaiX-image of the entire half cusp with Zeiss Software. The staining was conducted as described by Small et al. ${ }^{20}$

The other half of the cusp was directly assessed by microscopy at $\lambda=545 \mathrm{~nm}$ to detect cells expressing RFP.

\section{Histology and evaluation}

Thorough histological evaluation was conducted as previously described ${ }^{11}$ to assess extracellular matrix (ECM) integrity and cellular presence in the graft. One cusp per graft, including cusp, valvular wall, and pulmonary truncus, was fixed in formalin (4\%) and embedded in paraffin. Sections were cut with a thickness of $6 \mu \mathrm{m}$. While staining with hematoxylin and eosin (HE) was mainly performed as an overview and to analyze the number and localization of new cells in the graft, the Movat's pentachrome staining was conducted to analyze the ECM with its individual components (cell nuclei: brown; collagen: yellow; elastin: red; glycosaminoglycans: blue). A previously established repopulation score was used to compare the presence of cells within the interstitium of the two groups of TE valves. ${ }^{11}$

In addition, the degree of repopulation was assessed by staining a longitudinal section of $6 \mu \mathrm{m}$ with DAPI. After a MosaiX-image (Zeiss-Software) of the entire grafts was taken, the area with DAPI-positive nuclei was calculated in percentage to the complete longitudinal area. The adventitial tissue, as well as the anastomosis was not included in the evaluation.

Van Kossa staining was used to visualize mineralization occurring due to inflammation either by mechanical irritation or by remaining antigenicity.

\section{Immunofluorescence staining}

To identify cells in the graft at the time of sacrifice, one cusp per graft was embedded in a cryopreserved state in Tissue Tek ${ }^{\circledR}$ O.C.T. ${ }^{\mathrm{TM}}$, cut in cryosections of $6 \mu \mathrm{m}$ thickness, and stained against numerous epitopes by immunofluorescence staining as described previously. ${ }^{11}$

ECs were identified by antibodies against endothelial nitric oxide synthase (eNOS; 610297, 1:100; BD Biosciences) and von Willebrand Factor (vWF; A0082, 1:10.000; Dako). As the creation of a new endothelium is of great interest, we used a modified semiquantitative method by Baraki et al. to characterize the completeness of this superficial layer of cells positive for eNOS and vWF. ${ }^{12}$ The following three structures were assessed separately: surface of the cusp (I), of the valvular wall (II), and of the sinus (III) (absent: 0 , coverage $<50 \%$ : 1 , coverage $>50 \%$ : 2 , fully confluent: 3, maximum value: 12 ).

Antibodies against smooth muscle $\alpha$-actin (SMA, M0851, 1:50; Dako), myosin heavy chain 2 (MYH11; ab53219, 1:200; Abcam), and vimentin (M0725, 1:50; Dako) were used to identify interstitial cells. To assess the presence of immunological cells, antibodies against CD45 and CD8 were utilized.

While we used antibodies against collagen type I (C2456, 1:2000; Sigma-Aldrich) and collagen type IV (M0785, 1:25; Dako) to assess the ECM integrity, we also stained against intracellular procollagen type I to identify cells producing new matrix (52242, m-38-c, 1:10; Developmental Studies Hybridoma Bank, created by the NICHD of the NIH and maintained at the University of Iowa, Department of Biology, Iowa City, IA). Bound antibodies were visualized by $\mathrm{Cy} 3$-conjugated secondary antibodies directed against mouse or rabbit (715166-151 and 711-166-152, 1:200; Dianova), and nuclei were counterstained by DAPI (D1306, 1:15,000; Dianova).

Controls of the stainings are depicted in the Supplementary Figure S1 (Supplementary Data are available online at www.liebertpub.com/tec), including a positive control on and an isotype control in the concentration and immunoglobulin type of the utilized primary antibody.

\section{Statistical analysis}

Prism software was used to assess the graphs and to calculate the statistical significance by Student's $t$-test, and differences were considered relevant when $p<0.05$. All data are given in mean values and standard deviation.

\section{Results}

\section{Cultivation of ovine EC}

We were able to generate large numbers of EC from the peripheral blood of four sheep to use them for reendothelialization in the bioreactor. Those cells had the typical cobblestone morphology, proliferated rapidly, and were positive for vascular endothelial cadherin (VEcadherin) expression.

\section{Operation outcome}

The mean total operation time was $322.1 \pm 59.9 \mathrm{~min}$, including the mean aortic clamping time of $157.0 \pm 16.9 \mathrm{~min}$. All animals recovered from the anesthesia and were able to 
stand without assistance within $1 \mathrm{~h}$ after ending the inhalation of Isoflurane; except for one sheep with an EAV which died within $12 \mathrm{~h}$, leading to a reduction of animals in that group $(n=3)$. The cause of death could not be determined, but the valve showed good function at implantation and no damage to the graft could be identified at necropsy.

In the 12 months following implantation, the average weight increased from $85.1 \pm 8.8 \mathrm{~kg}$ to $98.6 \pm 8.9 \mathrm{~kg}$ (increase of $12.4 \pm 4.9 \mathrm{~kg}$ ). As the animals were at that age fully grown, the additional weight cannot be contributed to body growth but to the richer nutrition. In comparison to before, sheep did not experience any energy consuming gravidities and lactations during the experiment. One sheep (DAV) lost its incisor teeth, which led to weight loss. After proper care the sheep thrived again.

\section{Echocardiography}

Dimensions of the graft, as well as its function, were analyzed by transesophageal echocardiography. Measurement of the graft diameter revealed an increase in the EAV from $19.5 \pm 0.7$ to $25.0 \pm 1.7$, whereas the graft diameter of the DAV changes only marginally from $22.3 \pm 0.6$ to $23.0 \pm 0.0$. The valve orifice area of the EAV and DAV increased from $1.6 \pm 0.3 \mathrm{~cm}^{2}$ to $2.5 \pm 0.3 \mathrm{~cm}^{2}$ (significant, $p=0.02$ ) and from $1.7 \pm 0.3 \mathrm{~cm}^{2}$ to $2.3 \pm 0.5 \mathrm{~cm}^{2}$ (nonsignificant, $p=0.08$ ), respectively, with all EAVs and only one DAV increasing in orifice area. At the same time insufficiency did not increase, with two EAV grafts showing only mild insufficiency at sacrifice. Based on the fact that the valve orifice area did not decrease and as the mean, as well as the maximum gradient in general, did not increase in both groups, we can state that no signs of stenosis were observed.

Lower calculated orifice area at implantation was presumably caused by mild catecholamine support at weaning from the heart lung machine after the implantation thereby leading to accelerated flow across the implanted AV. Full data are given in Table 1.

\section{Morphological examination}

The heart as a whole did not appear hypertrophic or dilated after removing it from the thorax, and the graft showed well-adapted anastomoses. The luminal view on the cusps was possible by cutting the graft in longitudinal direction. Luminal surfaces of the graft revealed similar to the native adjacent truncus pulmonalis pigmented areas, very common for the particular sheep breed (black-headed meat sheep). Scattered small thrombotic nodules were found to some degree on all grafts especially on the ventricular surface. Macroscopically they could be identified in 8 out of 12 DAV cusps (Fig. 1A, B) and in 5 out of 9 EAV cusps (Fig. 1C, D). The proximal anastomosis was found to be very close to the proximal part of the cusps commissure. Otherwise cusps were translucent, thin, and showed no signs of deteriorations.

\section{Repopulation of the cusp surface with cells}

As visualized by phalloidin staining the ventricular cusp surface was repopulated to a greater extent than the arterial cusp surface. The ventricular cusp side was covered evenly with stained cells (green) (Fig. 2A, B); the arterial side,

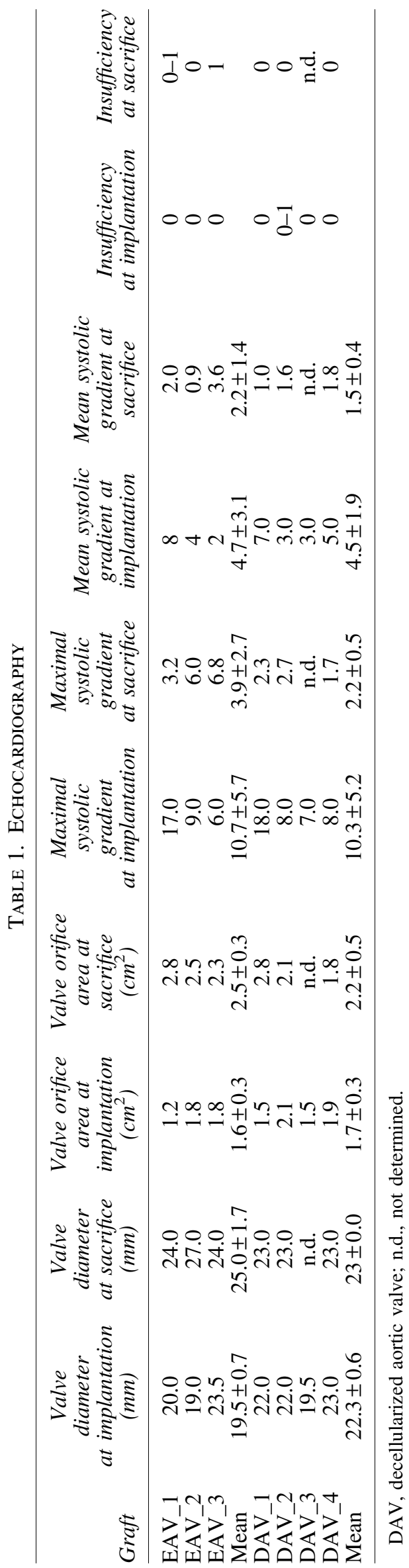



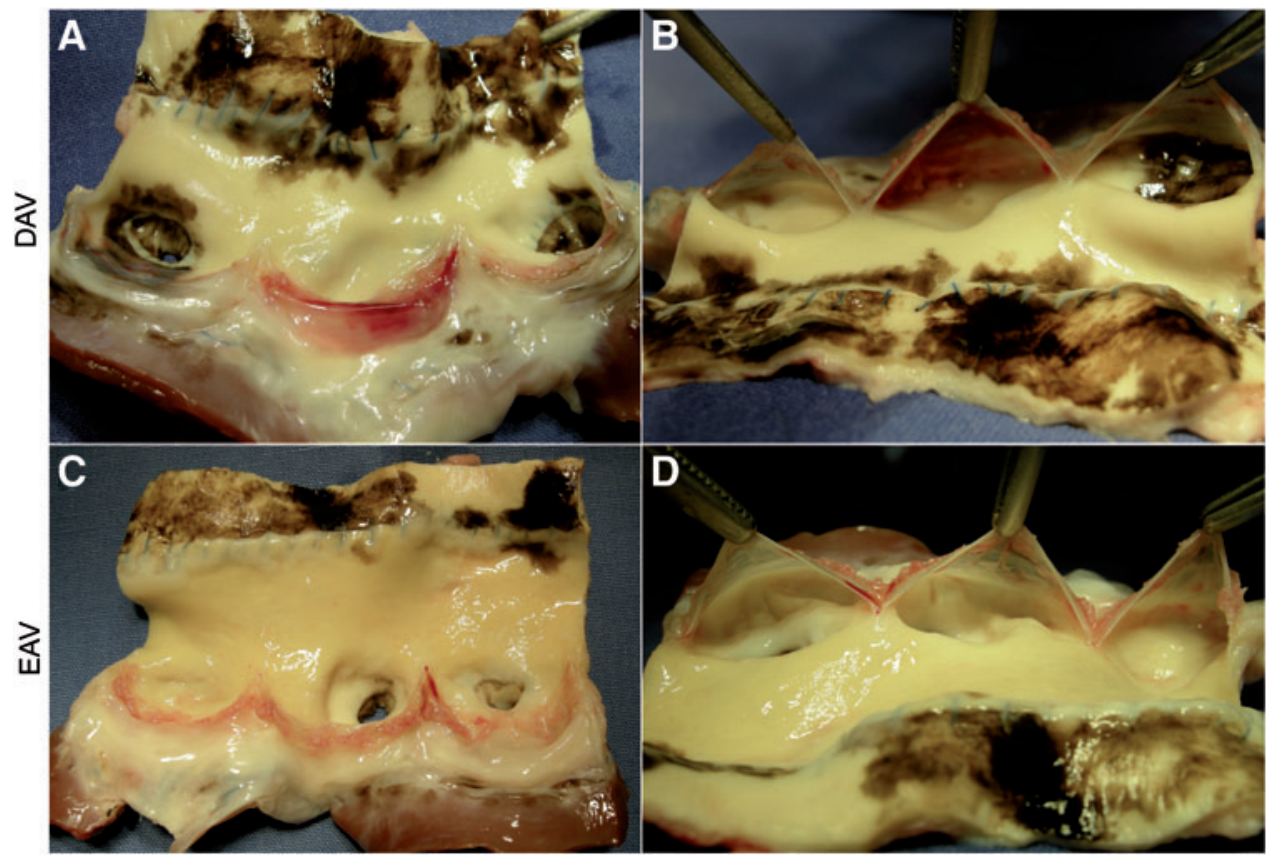

FIG. 1. Macroscopical examination of the explanted grafts. After explantation of DAVs $(\mathbf{A}, \mathbf{B})$ and EAVs $(\mathbf{C}, \mathbf{D})$, luminal view on the entire cusp $(\mathbf{A}, \mathbf{C})$ revealed well adapted anastomosis. The arterial view on the cusps $(\mathbf{B}, \mathbf{D})$ revealed thin cusps with small nodules on both surfaces, which otherwise lack additional signs of deterioration. DAVs, decellularized aortic valves. Color images available online at www.liebertpub.com/tec however, revealed discontinuous patches with cells (Fig. 2C, D). The repopulation of the ventricular surface was similar in both groups, with $62.2 \% \pm 26.1 \%$ for DAV cusps $(n=4)$ and $55.3 \% \pm 9.1 \%$ for EAV cusps $(n=3)$. The arterial cusp surface repopulation of EAVs $(52.0 \% \pm 18.0 \%)$ appeared to be more pronounced compared to DAVs $(22.3 \% \pm 16.6 \%)$, but the difference was not statistically significant (Fig. 3A).
When assessed for cells expressing red fluorescence, no positive cells could be identified.

\section{Examination of the matrix}

HE staining revealed cells present in the valvular walls and in the cusp. No difference could be observed concerning

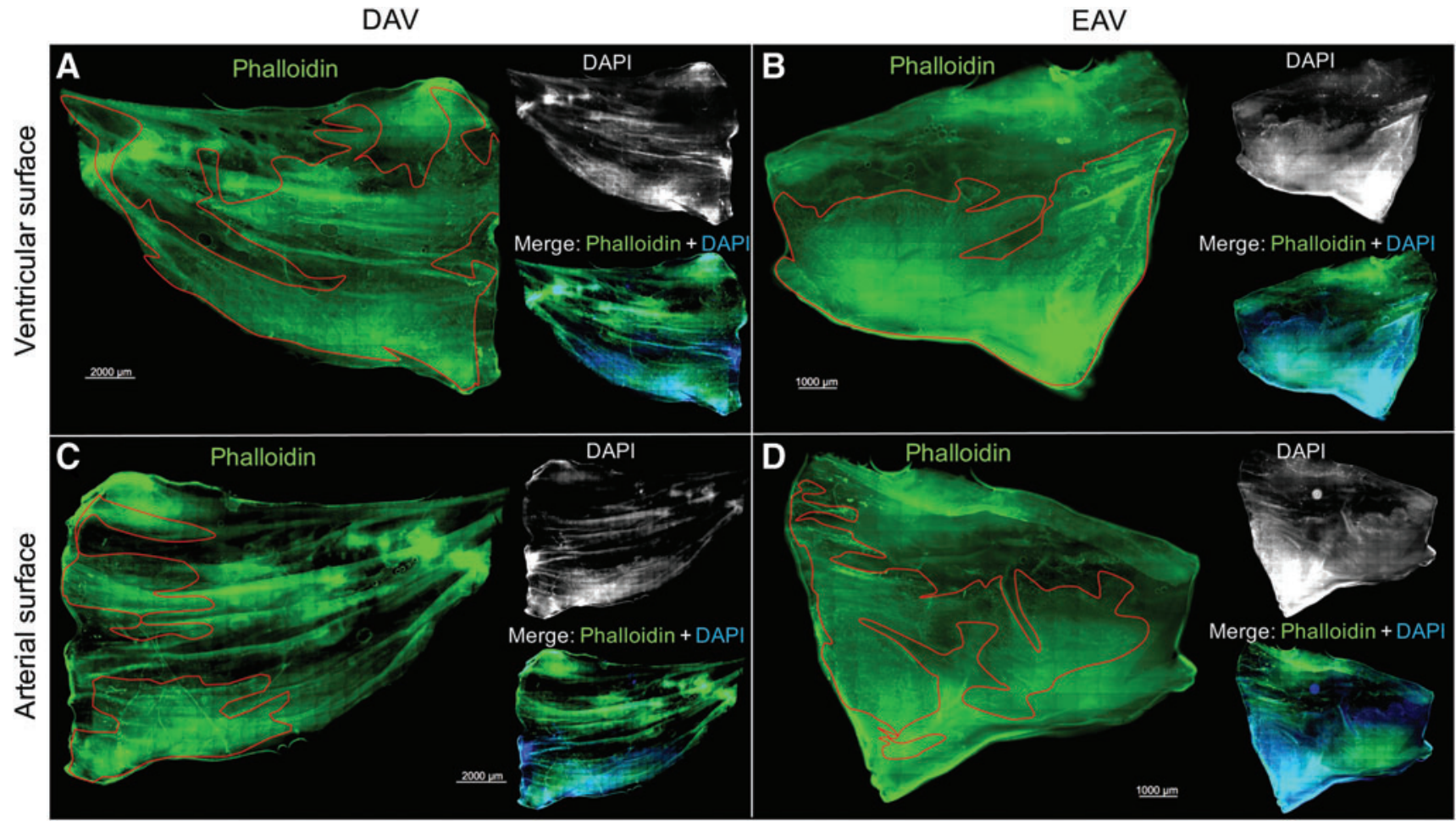

FIG. 2. Coverage of the cusp surface with cells stained by phalloidin and DAPI. The ventricular cusp surface was strongly covered by a continuous cell layer of both the DAV (A) and the EAV (B). The cell layer found on the arterial cusp surface of the DAV (C) and EAV (D) was smaller and appeared patchy (green, phalloidin; blue, DAPI; red, highlighted repopulated area). DAPI, 4',6-diamidino-2-phenylindole. Color images available online at www.liebertpub.com/tec 

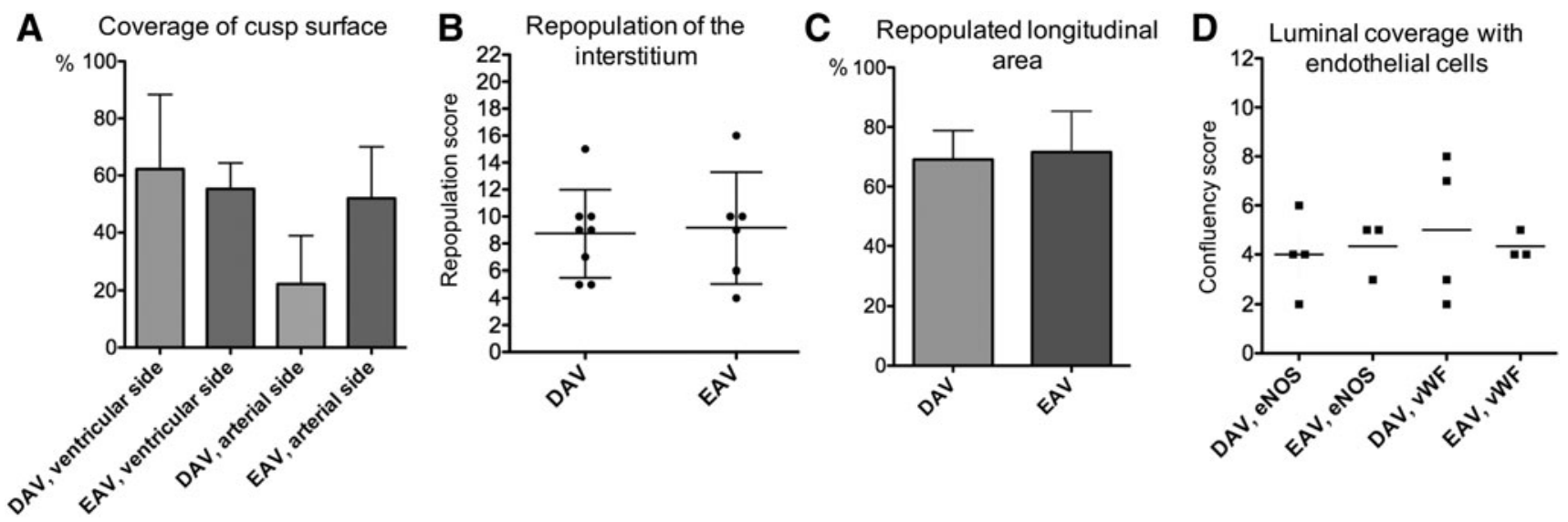

FIG. 3. Graft repopulation with cells. Three methods were used to measure the repopulation of the AVs with cells. The coverage of the cusp surface was analyzed by staining half a cusp with phalloidin, and the covered area was calculated in percentage of the complete grafts (A). No significant differences were found concerning cell density in longitudinal sections assessed by a scoring system (B) and measured as area containing nuclei (C). The luminal cell layer positive against the endothelial markers eNOS and vWF was found to be similarly confluent between DAV and EAV (D). AVs, aortic valves; eNOS, endothelial nitric oxide synthase; DAPI, 4',6-diamidino-2-phenylindole; vWF, von Willebrand factor.

the number and localization of cells between EAVs and DAVs. As seen by the phalloidin staining of cusps, cells were mainly concentrated on the ventricular surface at the proximal side. HE staining confirmed this finding, with cells also present in the interstitium proximal in the cusp (Fig. 4A), partly in the form of a neo-intimal hyperplasia (Supplementary Fig. S2A). Cells from the middle to the distal part of the cusps were found only on the surface. The distal part of the cusp was very thin, with the fibrosa being the main layer of the cusp. It was often covered with thrombi formations appearing light red in $\mathrm{HE}$ staining (Fig. 4B). Only a few cells were found on the arterial side, and no cells were found in the lamina fibrosa.

While the base of the cusp, which is directly adjacent to the proximal anastomosis, had a high cell concentration, the valvular wall was in general poorly repopulated. The cells in the valvular wall mainly localized in the adventitial part of the wall and the luminal part of the media being almost cell free (Fig. 4C). In general cell concentration decreased from proximal to distal. The presence of cells within the tissue of the two groups when assessed by the repopulation score was very similar and no graft could reach the highest score of 22 implying native-like cell density (Fig. 3B). Assessment of presence of nuclei in the longitudinal section revealed a repopulation of $70.1 \% \pm 10.6 \%$ of the section area and very similar degree of repopulation between DAV $(69.1 \% \pm 9.7 \%)$ and EAV (71.6\%土13.7\%) (Fig. 3C).

All three layers of the cusp were easily distinguishable when stained with pentachrome (Fig. 4D). The lamina spongiosa however was only visible in the proximal part of the cusp and the fibrosa dominated the cusp structure in bright yellow. Bright red, cell-free structures indicative for thrombi were found histologically on the cusp surface of six out of seven grafts (Fig. 4E and Supplementary Fig. S2B). Pentachrome staining revealed an intact ECM of the valvular wall, with collagen (yellow) being the dominant component, mixed with glycosaminoglycans (blue). Elastin fibers (red) were mainly in the luminal sided part of the graft, which was only poorly repopulated with cells, while the adventitial-sided part had a more blue coloration (Fig. 4F).

Big calcifications, appearing black after van Kossa staining, with a friable core were found in six out of seven grafts mainly in the proximal part of the graft near the proximal anastomosis and the cusp root (Supplementary Fig. S2C). Three out of seven grafts also bore small mineralization spots spread in an elsewise inconspicuous valvular wall matrix (Supplementary Fig. S2D). Both DAV and EAV were similarly affected. No black stained calcification foci were found in the cusp itself (Fig. 4H, I).

Immunofluorescence staining against collagen type I revealed that collagen was present in the complete cusp, but the lamina fibrosa showed a stronger staining (Fig. 5A). The fluorescence signal against collagen type I in the valvular wall showed a strong staining but a different orientation in the stronger repopulated adventitial-sided part of the media than in the luminal-sided poorly repopulated media (Fig. 5B). A thin layer was stained positive against collagen type IV on the arterial side of the cusp (Fig. 5C) and on the surface of the luminal wall (Fig. 5D). The signal of the ventricular cusp side was found to be multilayered especially on the proximal part (Fig. 5C). Positive staining was also found on vessel-like structures in the loose connective tissue surrounding the graft.

\section{Identification of repopulating cells}

Cells within and on the matrix were identified by immunofluorescence against specific cell markers. Cells on the luminal surface of the cusp and the valvular wall were found to be positive against vWF (Fig. 6A, B) and eNOS (Fig. 6C, D). Although the highest confluence of this new endothelium was found on the ventricular cusp surface, no complete confluence of vWF and eNOS positive cells for either the wall or the cusp was reached. Instead it appeared patchy, with some structures, such as the arterial cusp surface, having hardly any positive cells. Semiquantitative analysis of the coverage of the graft surface with cells positive for 


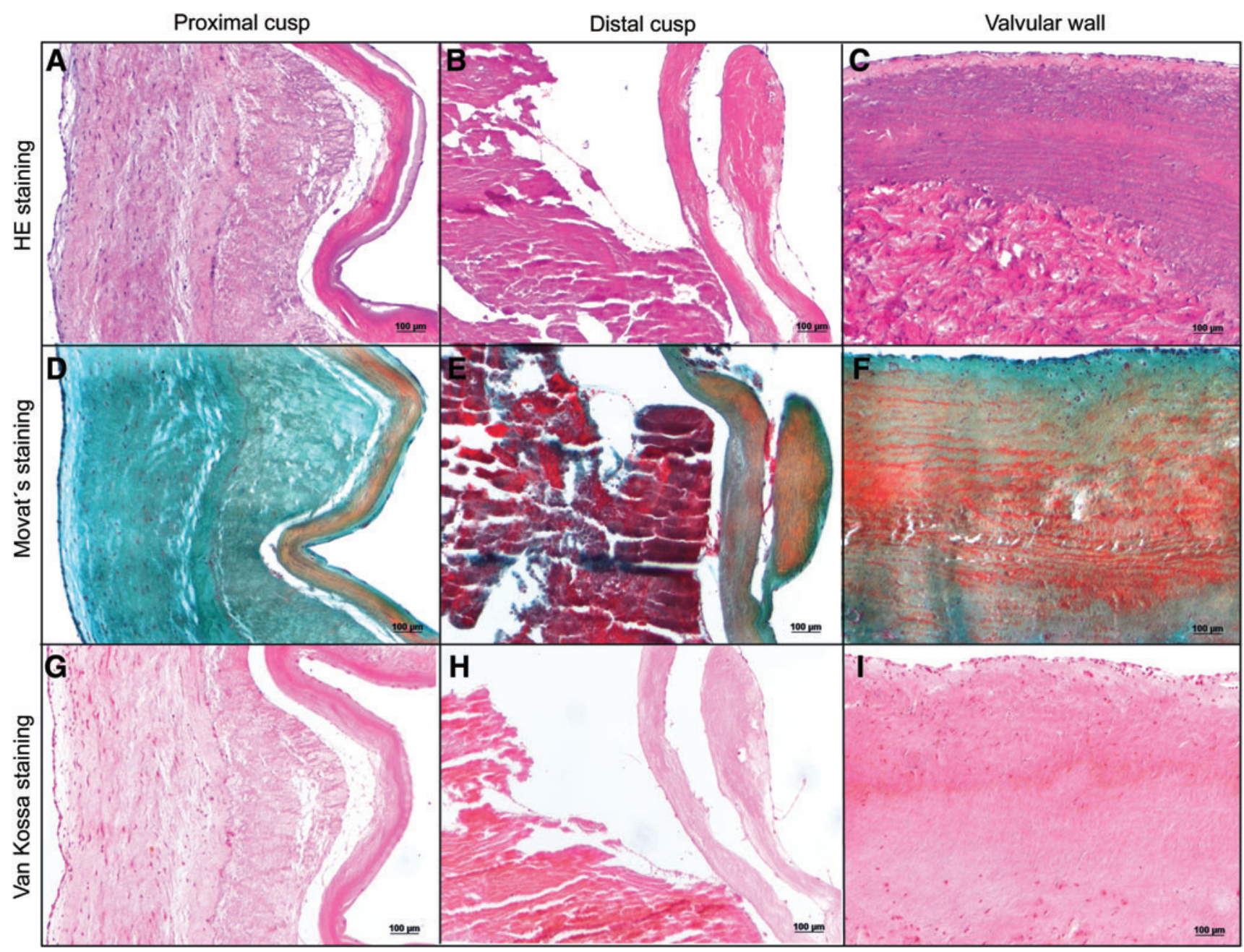

FIG. 4. Histological analysis of the explanted EAVs. HE staining of the graft revealed a strongly repopulated distal cusp (A), only superficial cells on the distal cusp (B) and a higher cell density in the adventitial-sided valvular wall (C) ( purple, nuclei; light red, cytoplasma and matrix). Movat's pentachrome staining showed blue coloration of the repopulated ventricular side on the proximal cusp (D). The strong yellow coloration of the lamina fibrosa with a red stained thrombus was found on the distal part of the cusp (E). In the valvular wall red fibrin fibrils were found mainly in the area with a small number of cells (F) (yellow, collagen; blue, GAGs; red, elastin; bright red, fibrin; brown, nuclei). No calcifications were found in this graft in the proximal (G) nor in the distal cusp (H) nor in the valvular wall (I) (black, mineralization; dark red, nuclei) (orientation: arterial side of cusp faces the right of the figure and the lumen of the valvular wall faces the top of the picture). HE, hematoxylin and eosin. Color images available online at www.liebertpub.com/tec

eNOS and vWF showed a very similar value for DAVs and EAVs, all grafts reached considerably lower values than the maximum possible of 12 points (Fig. 3D).

While repopulating cells in the entire graft were stained positive against vimentin (Fig. $6 \mathrm{E}, \mathrm{F}$ ), the majority of the cells in the cusp interstitium were also positive for SMA (Fig. 6G) and MYH11 (Fig. 6I). Within the valvular wall it was observed that less cells were positive for SMA (Fig. 6H) than vimentin and even less cells were positive against MYH11 (Fig. 6J). Cells stained positive for intracellular procollagen type I were found in the cusp interstitium (Fig. 6K), but a considerable number of cells positive for pro-collagen type I was found within the valvular wall (Fig. 6L). In the recipients' native pulmonary trunk hardly any cells positive for procollagen type I were found.

Cells positive for CD45, a pan-leukocyte marker, were detected in a moderate number distributed throughout the im- planted grafts in the cusp (Fig. 6M) and in the valvular wall (Fig. 6N). No specific structure was preferentially affected.

\section{Discussion}

Sheep at the age of 6 years show signs of aging, but at the same time retain several characteristics which render them a useful model for testing TE grafts

Following our previous work on PV replacement in 7year-old sheep, ${ }^{11}$ this study focused on two types of TE AV allografts. DAV and EAV were implanted in the aortic root position of adult sheep aged 6 years. Sheep used in this study showed age-related signs, such as loss of teeth, although it is difficult to translate the age of the animal directly to a corresponding age in humans.

We were able to obtain EC from the peripheral blood taken from the sheep, which is indicative for circulating 
FIG. 5. Analysis of the EAV matrix composition by immunofluorescence staining. Immunofluorescence staining against collagen type I stained the complete cusp with a stronger signal of the fibrosa (below on the picture) (A) and the complete valvular wall. (B) Staining against collagen type IV revealed a multilayered signal on the ventricular side of the cusp and a monolayered signal on the arterial side $(\mathbf{C})$. Positive staining was also found on the lumen of the valvular wall (above on the picture) (D). Blue, DAPI; red, stained epitope (orientation: ventricular side of the cusp and the lumen of the valvular wall face the top of the picture). Color images available online at www.liebertpub.com/ tec

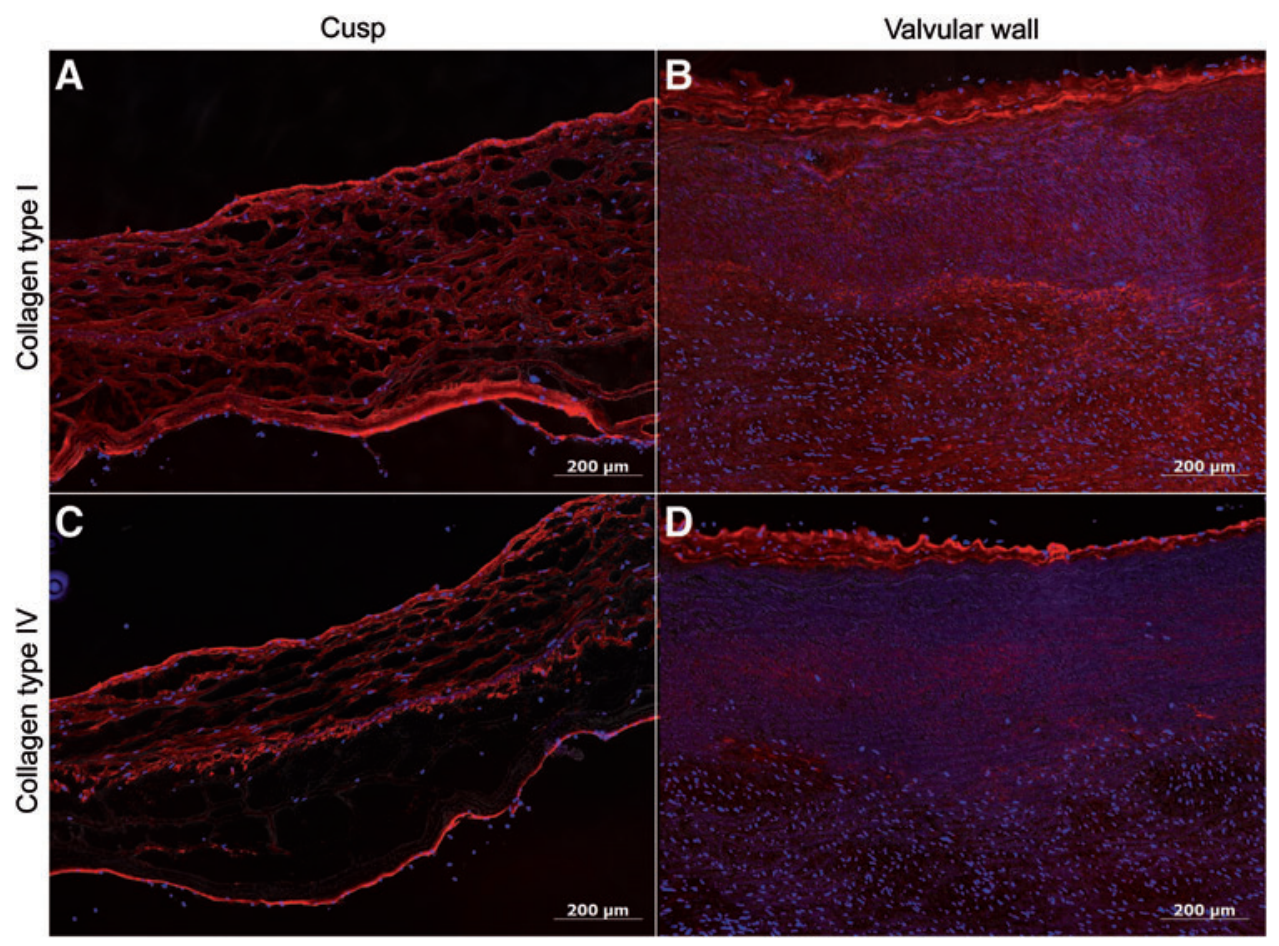

progenitor cells. Calcification spots were found at the base of the cusp and sporadically in the valvular wall, which were most likely linked to the anastomosis of the coronary ostia and to the proximal anastomosis, which in the aortic root position is very close to the cusp commissure. The base of the cusp is of course a very critical place to calcify as it would affect the valvular function most strongly. Due to the proximity of the observed mineralization to the anastomoses and found residual suture material, we assume that they are linked to the sutures.

Remaining cellular components, however, could also lead to an immunological reaction. Calcification is a distinct indication of immunological reaction, in particular, in sheep and is often used as a parameter to exclude bioprostheses with remaining antigenicity. ${ }^{21,22}$ In addition, calcification mechanisms in sheep resemble that in humans. ${ }^{23}$ Although an unfavorable observation, it shows that calcification processes in our animal model were not impaired. The generation of autologous ECs and the ability of calcification verify that even sheep of advanced age fulfill the properties that make it such a useful animal model for testing TE grafts.

\section{Compared to young animals the capacity to generate a new endothelium seems reduced, still signs of potential tissue regeneration do exist in 6-year-old adult sheep}

Decellularized PVs implanted in 7-year-old sheep have been compared with numerous publications on grafts implanted in the PV position in young animals. ${ }^{11}$ This comparison revealed that repopulation with the recipients' cells appeared reduced compared with published data. To accurately interpret the results from this current study, these need to be compared with studies focusing on TE grafts implanted in the aortic position of juvenile animals.

Gallo et al. reported finding spindle-shaped cells throughout the cusp interstitium when cellular AV allografts were im- planted in 12-month-old Vietnamese pigs for a period of 15 months. ${ }^{24}$ In our study, we found that interstitial cells were repopulating the cusp, but that even after 12 months in vivo only the distal and middle sections of the cusps had been partially repopulated with host's cells. While cells infiltrating the wall were identified as mostly fibroblasts, the ratio of cells positive for SMA was higher in the cusps. In native AV smooth muscle cells are found in the media of the arterial wall but not in the cusps. They are also found in high numbers in neointimal hyperplasia, which can lead to occlusion of vascular stents. ${ }^{25}$

Decellularized xenografts used for aortic root replacement in sheep aged between 4 and 9 months for up to 6 months in vivo in a study by Elkins et al. resulted in cusps being recellularized by up to $80 \% .^{26}$ Tudorache et al. reported finding endothelium on the aorta, sinus, and cusp, with EC covering the cusp up to the free margin and almost one third of the adventitial site being repopulated with procollagen type I positive cells in juvenile sheep (30$40 \mathrm{~kg}) 3$ months after AV replacement. ${ }^{19}$

Even though the cusp and valvular wall of the AVs in older sheep were partially repopulated, coverage with cells positive for endothelial markers and the density of cells positive for procollagen type I seemed to be reduced. A reason for this impaired ability to form a new endothelial layer could be the senescence of the adjacent native endothelium associated with increasing age. ${ }^{27}$ In contrast, the distribution of cells and cell identity was very similar to grafts implanted in juvenile animal models. $^{28,29}$ Besides the luminal coverage with EC, the adventitial part of the wall and the distal part of the cusp showed the highest cell density consisting of a mixture of fibroblasts, myofibroblasts, and smooth muscle cells. ${ }^{29}$

A possible regeneration potential in 6-year-old sheep is indicated (1) by the partial recellularization of the interstitium, (2) the partial re-endothelialization of the luminal graft surface, and (3) the production of matrix components by new cells. The regeneration of the DAV matrix in aged 


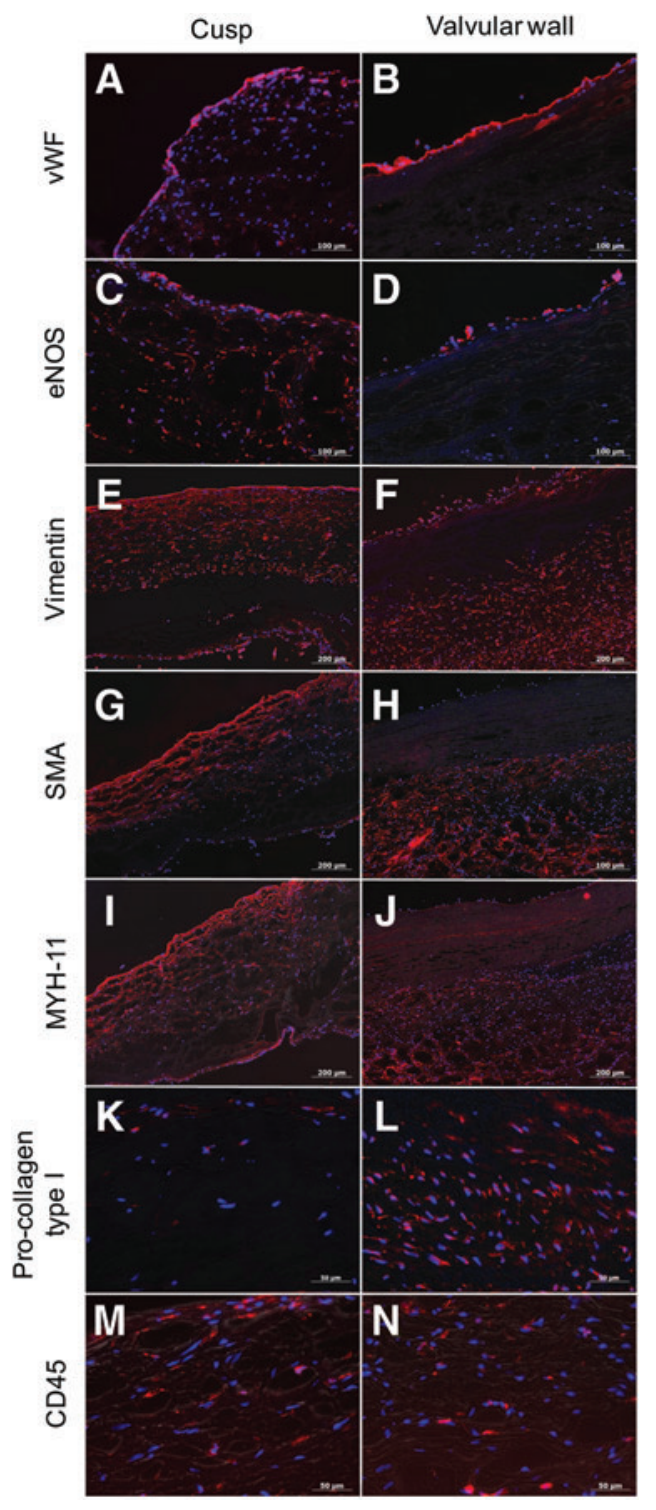

FIG. 6. Identification of repopulating cells in the EAV by immunofluorescence staining. vWF positive cells were found on the luminal surface of the cusp (A) and on the valvular wall (B). eNOS-positive cells were also found on the surface of the cusp (C) and on the valvular wall (D). All cells in the cusp (E) and in the wall (F) were positive for vimentin. While the majority of cells were positive for SMA in the cusp (G), only part was positive in the valvular wall $(\mathbf{H})$. When stained against MYH11 a similar observation was made, with the majority on the cells within the cusp being positive (I) but an even smaller number of positive cells were found within the valvular wall $(\mathbf{J})$. Few cells were stained positive for procollagen type in the cusp (K) but a high number of cells were stained in the valvular wall $(\mathbf{L})$. A moderate number of CD45-positive cells were found in the cusp (M) and in the valvular wall $(\mathbf{N})$. Blue, DAPI; red: stained epitope (orientation: ventricular side of the cusp and the lumen of the valvular wall face the top of the picture). MYH11, myosin heavy chain 2; SMA, smooth muscle $\alpha$-actin. Color images available online at www.liebertpub.com/tec sheep occurs to proceed in the same direction and pattern as that in younger sheep, but in a reduced or slower manner.

\section{The outcome of TE heart valves depends on the site of implantation}

Most studies of TE heart valves have been conducted on the $\mathrm{PV}$, despite the fact that the AV is the most frequently replaced heart valve. ${ }^{13}$ Studies using the PV cannot be taken as representative for the AV due to crucial differences between the two.

While the AV operates in a highly oxygenated highpressure environment, the PV functions in a low-pressure system with a low oxygen tension. ${ }^{14}$ The peak flow value in the AV position is $1.35 \pm 0.35 \mathrm{~m} / \mathrm{s},{ }^{30}$ whereas the peak velocity in the PV lies at $0.75 \pm 0.15 \mathrm{~m} / \mathrm{s} .^{31}$ In a study conducted by Angel et al. differences in proteins between AV and PV were detected, which were related to different oxygen tension and mechanical force. ${ }^{14}$ Each of these factors can potentially influence the outcome of graft function but also the repopulation of decellularized grafts. Even simple parameters such as the thickness of the valvular wall can influence the penetration depth of new cells in the tissue.

When measured, the valvular wall of DAV was found to be thicker compared with age-matched decellularized PV. ${ }^{29}$ Compared to our previous study on TE PV implanted in adult sheep, we observed differences in the degree of repopulation. While the arterial cusp surface of EAV had significantly stronger cell coverage, the repopulation of the interstitium was markedly stronger in the re-endothelialized PVs when using the same repopulation score.

\section{Re-endothelialization of decellularized grafts with autologous ECs did not prevent thrombi formation in adult sheep}

To prevent thrombotic events, we re-endothelialized the graft with EC obtained from the peripheral blood of the hosts. A previous study with re-endothelialized AVs based on decellularized grafts showed very promising results when implanted in young sheep, with no thrombotic nodules on the cusps and a complete endothelium 3 months after implantation. ${ }^{19}$ In our study, however, thrombotic nodules were also seen on the re-endothelialized cusps and we were unable to detect a complete endothelium, even at 12 months after implantation. In addition to the difference in age, the source of the EC also differed in the studies. While we used EC from peripheral blood, Tudorache et al. used ECs obtained from the autologous jugular vein. ${ }^{19}$ ECs are known to exhibit different properties depending on their localization. ${ }^{32}$ In this context it is possible that EC taken from peripheral blood do not exhibit full antithrombotic properties, especially when taken from older individuals. However, removing a vessel from elderly multimorbid patients in the clinical setting is not desirable.

Seeded cells, expressing RFP, could not be detected 12 months after implantation. Cells may have silenced the RFP-expression upon implantation, or, more likely, seeded RFP cells were replaced by other ECs.

Considering that the DAVs resulted in a similarly good outcome to EAVs, re-endothelialization may not be necessary, as the time-consuming and invasive procedure of generating EAVs negates possible benefits in older patients. An antithrombotic surface of AV substitutes is essential, especially as this is characterized as a great asset of biological 
grafts. Thrombi on the AV could cause occlusions of vessels and in the worst case coronary thrombosis leading to cardiac infarction. Our results indicate that the surface of the EAV and DAV bears some thrombogenic potential. The small thrombotic alterations found only on the cusps suggest, however, that patients would need anticoagulant therapy over a longer period than used in this study but in a lower dosage than required for mechanical valves. This could be addressed in a follow-up study.

It is also possible that not all cells cultivated on the cusp remained there after implantation. While the number of EC used for re-endothelialization was comparable to that used in the study in young sheep, the flow through the valve in adult sheep with approximately double the weight is higher, and EC cultivated in the bioreactor system to a flow of $1 \mathrm{~L} /$ min through the valve now require training up to a more physiological flow of 2 or even $3 \mathrm{~L} / \mathrm{min}$ to withstand the shear stress after implantation.

\section{AV grafts show adaptation in size as well}

Echocardiographical results revealed good function 12 months after implantation, with only two EAV having developed a mild but clinically irrelevant regurgitation. Although the diameter and orifice area increased in some grafts, insufficiency did not occur, at least not to the expected degree. Due to a lack of suitable grafts for adult sheep, we must postulate retrospectively that some allografts may have been too small for the animal size. The results, however, show that allografts based on decellularized matrices can adapt in size to some degree without loss of function. Even when the valvular wall dilates, there is still co-optation of the cusp, showing that undersized grafts can adapt to a gap in size.

To conclude, TE allografts based on decellularized matrix show good functional properties in aged sheep, but reendothelialization did not improve the outcome and longer anticoagulant therapy is required. Signs of regenerative potential of older individuals are noticeable in grafts even when implanted in the AV position.

\section{Acknowledgments}

The authors are grateful for the excellent technical assistance of Klaus Höffler, Astrid Diers-Kettekat, Rosalinde Katt, Slavica Schümann, Karin Peschel, Doreen Unger, and Petra Ziehme. The authors also thank Nina McGuiness, ELS, for the editorial revision. This work was funded by the Cortiss Foundation and by the German Research Foundation (Deutsche Forschungsgemeinschaft, DFG) through the Cluster of Excellence "From regenerative biology to reconstructive therapy" (REBIRTH).

\section{Disclosure Statement}

No competing financial interests exist.

\section{References}

1. Shan, L., Saxena, A., McMahon, R., Wilson, A., and Newcomb, A. A systematic review on the quality of life benefits after aortic valve replacement in the elderly. $\mathrm{J}$ Thorac Cardiovasc Surg 145, 1173, 2013.
2. Cebotari, S., Tudorache, I., Ciubotaru, A., et al. Use of fresh decellularized allografts for pulmonary valve replacement may reduce the reoperation rate in children and young adults: early report. Circulation 124, S115, 2011.

3. Neumann, A., Cebotari, S., Tudorache, I., Haverich, A., and Sarikouch, S. Heart valve engineering: decellularized allograft matrices in clinical practice. Biomed Tech (Berl) 58, 453, 2013.

4. Hammermeister, K.E., Sethi, G.K., Henderson, W.G., Oprian, C., Kim, T., and Rahimtoola, S. A comparison of outcomes in men 11 years after heart-valve replacement with a mechanical valve or bioprosthesis. Veterans Affairs Cooperative Study on Valvular Heart Disease. N Engl J Med 328, 1289, 1993.

5. Zhao, D.F., Seco, M., Wu, J.J., et al. Mechanical versus bioprosthetic aortic valve replacement in middle-aged adults: a systematic review and meta-analysis. Ann Thorac Surg 102, 315, 2016.

6. Birkmeyer, N.J., Birkmeyer, J.D., Tosteson, A.N., Grunkemeier, G.L., Marrin, C.A., and O'Connor, G.T. Prosthetic valve type for patients undergoing aortic valve replacement: a decision analysis. Ann Thorac Surg 70, 1946, 2000.

7. Arias, E. United States life tables, 2010. Natl Vital Stat Rep 63, $1,2014$.

8. Iung, B., Baron, G., Butchart, E.G., et al. A prospective survey of patients with valvular heart disease in Europe: the Euro Heart Survey on Valvular Heart Disease. Eur Heart J 24, 1231, 2003.

9. Efimenko, A.Y., Kochegura, T.N., Akopyan, Z.A., and Parfyonova, Y.V. Autologous stem cell therapy: how aging and chronic diseases affect stem and progenitor cells. Biores Open Access 4, 26, 2015.

10. Ju, Z., and Rudolph, K.L. Telomeres and telomerase in stem cells during aging and disease. Genome Dyn 1, 84, 2006.

11. Theodoridis, K., Tudorache, I., Calistru, A., et al. Successful matrix guided tissue regeneration of decellularized pulmonary heart valve allografts in elderly sheep. Biomaterials 52, 221, 2015.

12. Baraki, H., Tudorache, I., Braun, M., et al. Orthotopic replacement of the aortic valve with decellularized allograft in a sheep model. Biomaterials 30, 6240, 2009.

13. Beckmann, A., Funkat, A.K., Lewandowski, J., et al. Cardiac surgery in Germany during 2014: a report on behalf of the german society for thoracic and cardiovascular surgery. Thorac Cardiovasc Surg 63, 258, 2015.

14. Angel, P.M., Nusinow, D., Brown, C.B., et al. Networkedbased characterization of extracellular matrix proteins from adult mouse pulmonary and aortic valves. J Proteome Res 10, 812, 2011.

15. Aper, T., Teebken, O.E., Kruger, A., Heisterkamp, A., Hilfiker, A., and Haverich, A. Development of implantable autologous small-calibre vascular grafts from peripheral blood samples. Zentralbl Chir 138, 173, 2013.

16. Vukadinovic-Nikolic, Z., Andree, B., Dorfman, S.E., et al. Generation of bioartificial heart tissue by combining a three-dimensional gel-based cardiac construct with decellularized small intestinal submucosa. Tissue Eng Part A 20, 799, 2014.

17. Lichtenberg, A., Cebotari, S., Tudorache, I., et al. Flowdependent re-endothelialization of tissue-engineered heart valves. J Heart Valve Dis 15, 287, 2006.

18. Lichtenberg, A., Tudorache, I., Cebotari, S., et al. In vitro re-endothelialization of detergent decellularized heart valves under simulated physiological dynamic conditions. Biomaterials 27, 4221, 2006. 
19. Tudorache, I., Calistru, A., Baraki, H., et al. Orthotopic replacement of aortic heart valves with tissue-engineered grafts. Tissue Eng Part A 19, 1686, 2013.

20. Small, J., Rottner, K., Hahne, P., and Anderson, K.I. Visualising the actin cytoskeleton. Microsc Res Tech 47, 3, 1999.

21. Wu, S., Liu, Y.L., Cui, B., Qu, X.H., and Chen, G.Q. Study on decellularized porcine aortic valve/poly (3-hydroxybutyrateco-3-hydroxyhexanoate) hybrid heart valve in sheep model. Artif Organs 31, 689, 2007.

22. Brizard, C.P., Brink, J., Horton, S.B., Edwards, G.A., Galati, J.C., and Neethling, W.M. New engineering treatment of bovine pericardium confers outstanding resistance to calcification in mitral and pulmonary implantations in a juvenile sheep model. J Thorac Cardiovasc Surg 148, 3194, 2014.

23. Schoen, F.J., Hirsch, D., Bianco, R.W., and Levy, R.J. Onset and progression of calcification in porcine aortic bioprosthetic valves implanted as orthotopic mitral valve replacements in juvenile sheep. J Thorac Cardiovasc Surg 108, 880, 1994.

24. Gallo, M., Bonetti, A., Poser, H., et al. Decellularized aortic conduits: could their cryopreservation affect postimplantation outcomes? A morpho-functional study on porcine homografts. Heart Vessels 31, 1862, 2016.

25. Murphy, J., and Boyle, F. Predicting neointimal hyperplasia in stented arteries using time-dependant computational fluid dynamics: a review. Comput Biol Med 40, 408, 2010.

26. Elkins, R.C., Goldstein, S., Hewitt, C.W., et al. Recellularization of heart valve grafts by a process of adaptive remodeling. Semin Thorac Cardiovasc Surg 13, 87, 2001.

27. Ross, M.D., Malone, E., and Florida-James, G. Vascular ageing and exercise: focus on cellular reparative processes. Oxid Med Cell Longev 2016, 3583956, 2016.

28. Tudorache, I., Theodoridis, K., Baraki, H., et al. Decellularized aortic allografts versus pulmonary autografts for aortic valve replacement in the growing sheep model: haemodynamic and morphological results at 20 months after implantation. Eur J Cardiothorac Surg 49, 1228, 2016.

29. Della Barbera, M., Valente, M., Basso, C., and Thiene, G. Morphologic studies of cell endogenous repopulation in decellularized aortic and pulmonary homografts implanted in sheep. Cardiovasc Pathol 24, 102, 2015.

30. Otto, C.M. Clinical practice. Evaluation and management of chronic mitral regurgitation. N Engl J Med 345, 740, 2001.

31. Oh, J.K., Appleton, C.P., Hatle, L.K., Nishimura, R.A., Seward, J.B., and Tajik, A.J. The noninvasive assessment of left ventricular diastolic function with two-dimensional and Doppler echocardiography. J Am Soc Echocardiogr 10, 246, 1997.

32. Corada, M., Morini, M.F., and Dejana, E. Signaling pathways in the specification of arteries and veins. Arterioscler Thromb Vasc Biol 34, 2372, 2014.

Address correspondence to: Andres Hilfiker, PhD

Leibniz Research Labs for Biotechnology and Artificial Organs (LEBAO)

Hannover Medical School Carl Neuberg Str. 1 D-30625 Hannover Germany

E-mail: hilfiker.andres@mh-hannover.de

Received: April 6, 2017

Accepted: June 8, 2017

Online Publication Date: November 3, 2017 